\title{
The Gap Sampler: A Versatile Microfluidic Autosampler for Electrospray Ionization Mass Spectrometry
}

\author{
Jérôme Kaeslin\$ ${ }^{*}$ \\ §SCS-Metrohm Award for the Best Oral Presentation in Analytical Sciences
}

\begin{abstract}
Microfluidic autosamplers for electrospray ionization mass spectrometry (ESI-MS) are of major importance when using ESI-MS as a high-throughput and low sample consumption analytical method. In this article, microfluidic ESI-MS autosampler designs are overviewed and a group-owned prototype is discussed. The socalled gap sampler is a pin-based sampler for miniaturized flow injection (FI) analysis. To date, it has been used in various applications. Following proof of concept applications with FI of small molecules, pin modifications were implemented for unspecific and specific extraction for the analysis of complex samples. Most recently, further optimization allowed the study of non-covalent protein-ligand interactions for bioaffinity screenings, which constitutes a major milestone in the development of this novel high-throughput autosampler.
\end{abstract}

Keywords: Autosampler · Extraction · Mass Spectrometry $\cdot$ Microfluidics $\cdot$ Screening

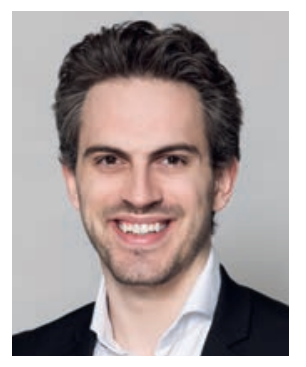

Jérôme Kaeslin studied chemistry at ETH Zurich. He undertook his Master studies as a Werner Siemens fellow and finished in 2018 as a Willi Studer Prize winner. For his Master thesis, he stayed at the Université de Genève in the group of Prof. Gérard Hopfgartner to learn about the retention time prediction of metabolites for LC-MS analysis. He is now pursuing his $\mathrm{PhD}$ studies in the group of Prof. Renato Zenobi back at ETH Zurich. There, he is supported by the Scholarship Fund of the Swiss Chemical Industry (SSCI) and focuses on the clinical and pharmaceutical applications of ESI-MS.

\section{ESI-MS Autosampler Miniaturization}

Speed, sensitivity and specificity are among a chemist's main criteria when selecting an analytical method. Mass spectrometry (MS) is a technique which performs outstandingly well in these aspects. ${ }^{[1]}$ In combination with electrospray ionization (ESI), complex molecules can be rapidly and gently ionized followed by a transfer into a mass spectrometer. ${ }^{[2]}$ Hence, ESI-MS is often utilized for high-throughput analysis of pharmaceutically relevant molecules, e.g. in metabolic fingerprinting, ${ }^{[3]}$ screening for drug discovery ${ }^{[4]}$ or quality assessment of combinatorial libraries. ${ }^{5]}$ Typically, the sample handling is significantly slower than the MS acquisition itself. As a consequence, low speed analyte introduction often constrains a method's throughput. ${ }^{[1]}$ To fully exploit the strengths of MS, it is important to rapidly supply the instrument with sample, while minimizing the sample consumption. Therefore, developments in the past years were focused on an automated microfluidic sample handling upstream to ESI-MS. In this article, an overview of microfluidic autosamplers from other researchers is provided and our group-owned prototype is discussed.

*Correspondence: J. Kaeslin, E-mail: jerome.kaeslin@org.chem.ethz.ch ETH Zurich, Vladimir-Prelog-Weg 3, CH-8093 Zurich, Switzerland
The classical types of ESI sample delivery are direct infusion (DI) or flow injection (FI). ${ }^{6]}$ For DI, one sample at a time is delivered into the ESI capillary at a flow rate of a few microliters per minute with a syringe pump. Whereas for FI, a sample plug is loaded into a carrier liquid which transports the analytes towards the ESI source. Automated FI typically requires a few microliters of sample and achieves a cycle time of $\approx 30 \mathrm{~s}$. By using nanoliter injection valves, the sample volume can be decreased down to $4 \mathrm{nl} .^{[7]}$ Likewise, a cycle time down to $\approx 6 \mathrm{~s}$ was reported by operating multiple injectors in parallel or series. ${ }^{[8]}$ To improve speed and reduce sample consumption, multiple research groups tried to miniaturize DI or FI. Felten et al. reported a mini-DI system where the sample is aspirated directly from a multiwell plate with the ESI capillary.[6] As little as $120 \mathrm{nl}$ of sample were consumed with cycle times of less than 10 s. Somewhat similarly, Pei et al. aspirated air-segmented samples from a multiwell plate. ${ }^{[9]}$ Partitioned by air, up to hundred $10 \mathrm{nl}$ sample plugs were loaded into a capillary. Then, the plugs were pushed towards a nanospray source resulting in a $1.5 \mathrm{~s}$ cycle time. Jin et al. reported an easyto-fabricate mini-FI autosampler ${ }^{[10]}$ in which $\approx 3-6 \mathrm{nl}$ of sample were picked up by spontaneous injection through an opening in a bent ESI capillary. Subsequently, the sample plug was transported to the ESI emitter by a carrier liquid reaching a $\approx 21 \mathrm{~s}$ cycle time.

The NanoMate from Advion is a commercial mini-DI system. ${ }^{[11]}$ With this device, $1-20 \mu$ of sample are aspirated from a microwell plate with a disposable pipette. Next, the sample is conveyed from the pipette into an opening of a multi-nozzle nano-

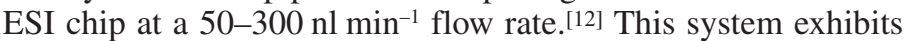
a cycle time of $\approx 40 \mathrm{~s}$ and is free of carry over because the pipette tips and the nozzles are single-use. ${ }^{[13]}$ Another commercial system is the RapidFire merchandised by Agilent. ${ }^{[14]}$ It includes a solidphase extraction step for purification between the sample pickup and the delivery to the ESI-source. This makes the autosampler ideal for enzyme inhibition assays, because interfering components are removed before introduction into the ESI source. Typical sample volumes are $1-5 \mu \mathrm{l}$ at a $4-5$ s cycle time.

Additionally, there are microfluidic autosamplers which do not classify as miniaturized DI or FI. Shimadzu commercialized a 
probe ESI (PESI) system which can pick up $\approx 3$ pl of sample every $\approx 0.4 \mathrm{~s}$ with a solid pin from a multiwell plate. ${ }^{[15]}$ Subsequently, the liquid is electrosprayed by application of a high voltage directly to the pin. Yet another approach is based on the ionization of droplets originating from an acoustic mist generator. ${ }^{[16]}$ This setup achieves a $\approx 0.4$ s cycle time and consumes $\approx 15 \mathrm{nl}$ of sample.

\section{The Gap Sampler as a Mini-FI Autosampler}

The autosampler shown in Fig. 1a was originally developed by Hoffman La-Roche AG. ${ }^{[17]}$ In a collaboration, our group refined the device and developed novel applications. ${ }^{[18]}$ The key components of the autosampler are two linearly arranged consecutive feed and drain capillaries embedded inside a pressure chamber as shown in Fig. 1b. A few microliters per minute of carrier liquid are supplied by the fused silica feed capillary via a syringe pump. The liquid bridges $\mathrm{a} \approx 400 \mu \mathrm{m}$ gap between the feed and the stainless steel drain capillary. $\mathrm{A} \approx 30$ mbar overpressure inside the chamber prevents flooding. By applying a high voltage to the drain capillary, the liquid is constantly electrosprayed.

The liquid bridge is used as an introduction system for FI. This is realized by a $356 \mu \mathrm{m}$ diameter metal pin held by a robot arm. The pin picks up a few nanoliters of sample from a multiwell plate, enters the pressure chamber through a sealing port and injects the nanodroplet into the liquid bridge as schematically shown in Fig. 1c. Subsequently, the pin is washed with two solvent mixtures, dried by nitrogen and reinitialized for the next injection. In a first study by Neu et al., the FI characteristics were studied using cortisone (1) as a model analyte and a 50:50 MeOH/ $\mathrm{H}_{2} \mathrm{O} V / \mathrm{V}$ carrier liquid as shown in Fig. 2a. ${ }^{[18]}$ Cycle times down to $15 \mathrm{~s}$ and $\approx 10 \mathrm{nl}$ injection volumes were reported. Additionally, less than $1 \%$ sample carryover was determined. Moreover, a linear response between injected analyte concentration and detected signal was demonstrated as shown in Fig. 2 b for 1. Importantly, a major finding was that the liquid bridge exhibits a certain self-
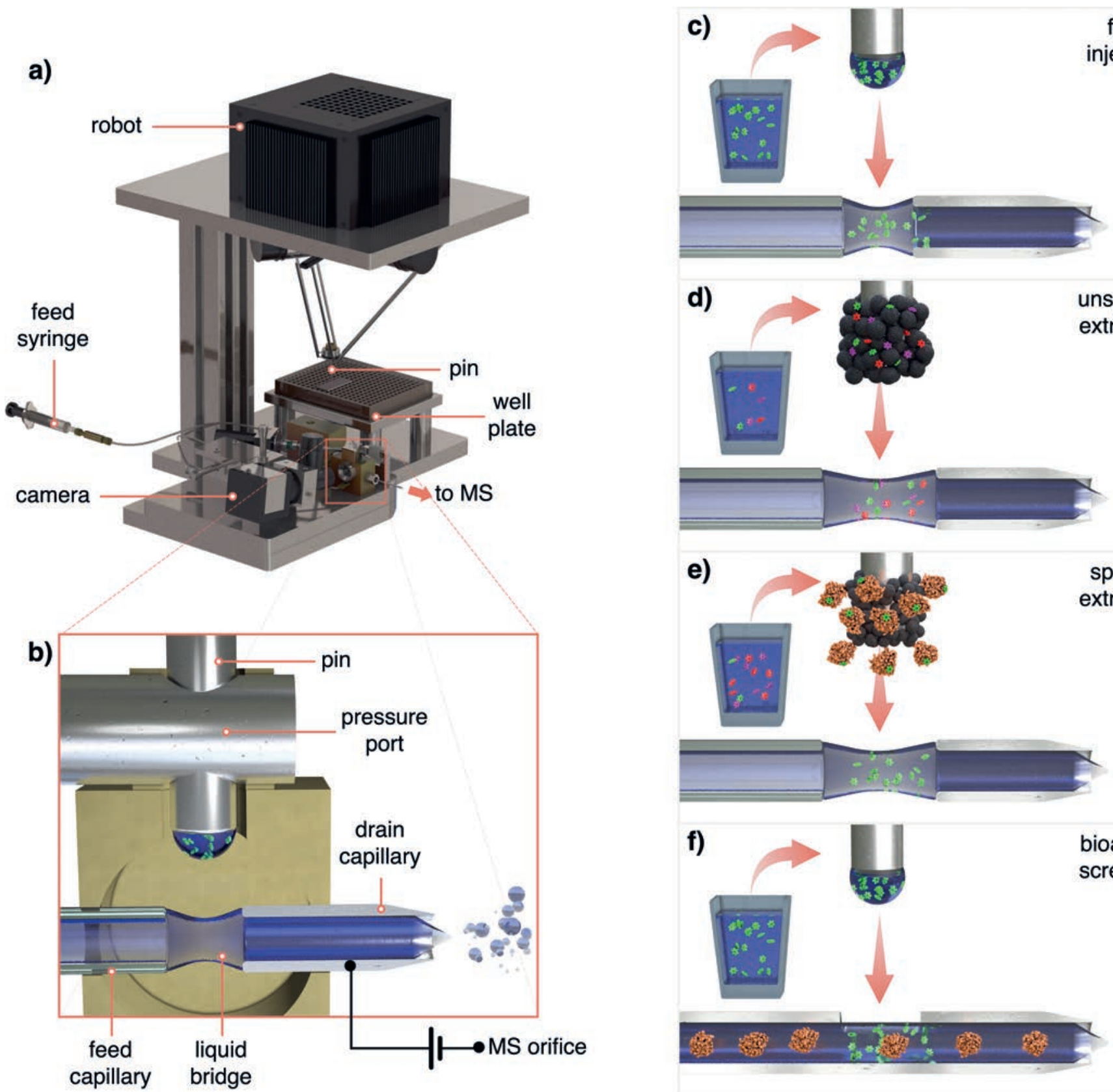

flow injection

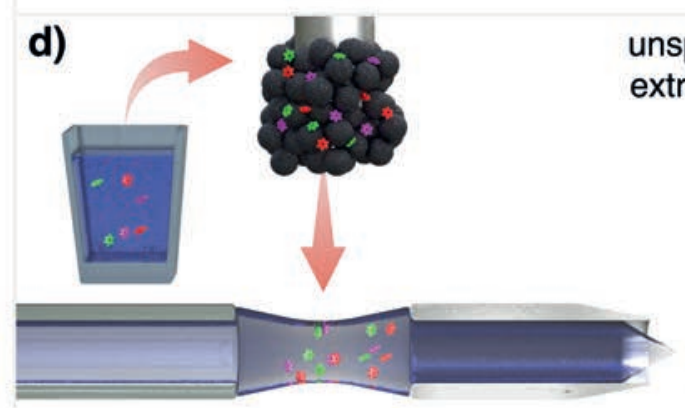

unspecific

e)

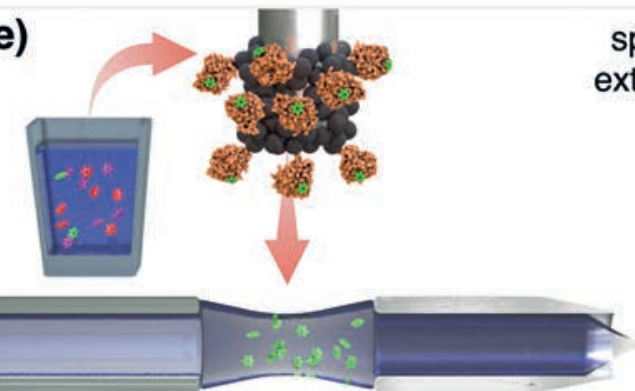

specific cific

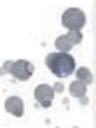
xtraction

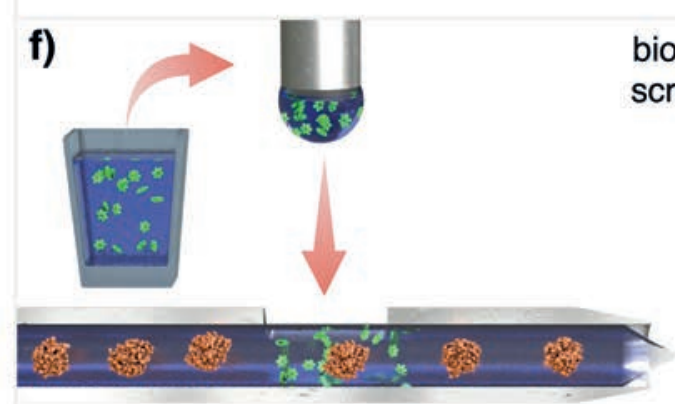

bioaffinity screening

Fig. 1. Overview of the gap sampler and its applications. a) representation of the autosampler; b) schematic zoom into the pressure chamber during injection; c) flow injection: a sample is picked-up with the pin from a well and injected into the carrier liquid; d) unspecific extraction: analytes are extracted with $\mathrm{a}_{18}$ solid-phase on the pin and eluted by the carrier liquid. e) specific extraction: one analyte is specifically extracted with an immobilized protein and eluted by the carrier liquid. f) bioaffinity screening: a ligand is injected into a stream of protein target. Detection of the ligand-protein complex indicates binding. 
stabilizing effect under a regime of defined pressure, flow rate and gap distance.

In a follow-up study, Neu et al. benchmarked the gap sampler's FI characteristics with a $10 \mathrm{nl}$ microvalve. ${ }^{[19]}$ In terms of flush-out profiles, the gap sampler and the microvalve perform equally well. The cause of the gap sampler's peak broadening of the flush-out profiles was attributed to laminar flow dead-zones and longitudinal diffusion. However, by using larger inner diameter feed and drain capillaries, narrower flush-out profiles were achieved and the microvalve was outperformed.

\section{The Gap Sampler for Automated Extraction}

In analytical chemistry, a common kind of sample preparation prior to analysis is solid-phase microextraction (SPME). In this extraction process, analytes are enriched in a small volume of a solidphase extractant material and subsequently desorbed for analysis. ${ }^{[20]}$ SPME is particularly helpful to remove ESI incompatible constituents. The gap sampler can be utilized to automate this process. Instead of using the bare solid pin for injection, it can be functionalized with a solid-phase extraction material. Ghiasikhou et al. functionalized the pin by immobilizing octadecyl $\left(\mathrm{C}_{18}\right)$ modified silica particles. ${ }^{[21]}$ Thus, automated SPME extraction was performed by (I) immersing the pin for a defined time into the sample solution, (II) quickly washing off unspecific analytes from the wetted surface and (III) immersing the pin into the liquid bridge as shown in Fig. 1d. By using 80:20 ACN/ $/ \mathrm{H}_{2} \mathrm{O} V / V+0.1 \% \mathrm{~V} / \mathrm{V}$ formic acid as a carrier liquid, the analytes are desorbed in less than $25 \mathrm{~s}$. To assess the extraction's performance, $500 \mathrm{ng} \mathrm{ml}^{-1}$ nordazepam (2) in phosphate-buffered saline (PBS) was extracted. Fig. 3 shows how the signal intensity increases with longer extraction time. $\mathrm{A} \approx 9$-fold signal rise is observed when the extraction equilibrium is reached after $\approx 30 \mathrm{~min}$. To keep the analysis time short, it is advantageous to stir the pin in the sample and to extract under a kinetically limited
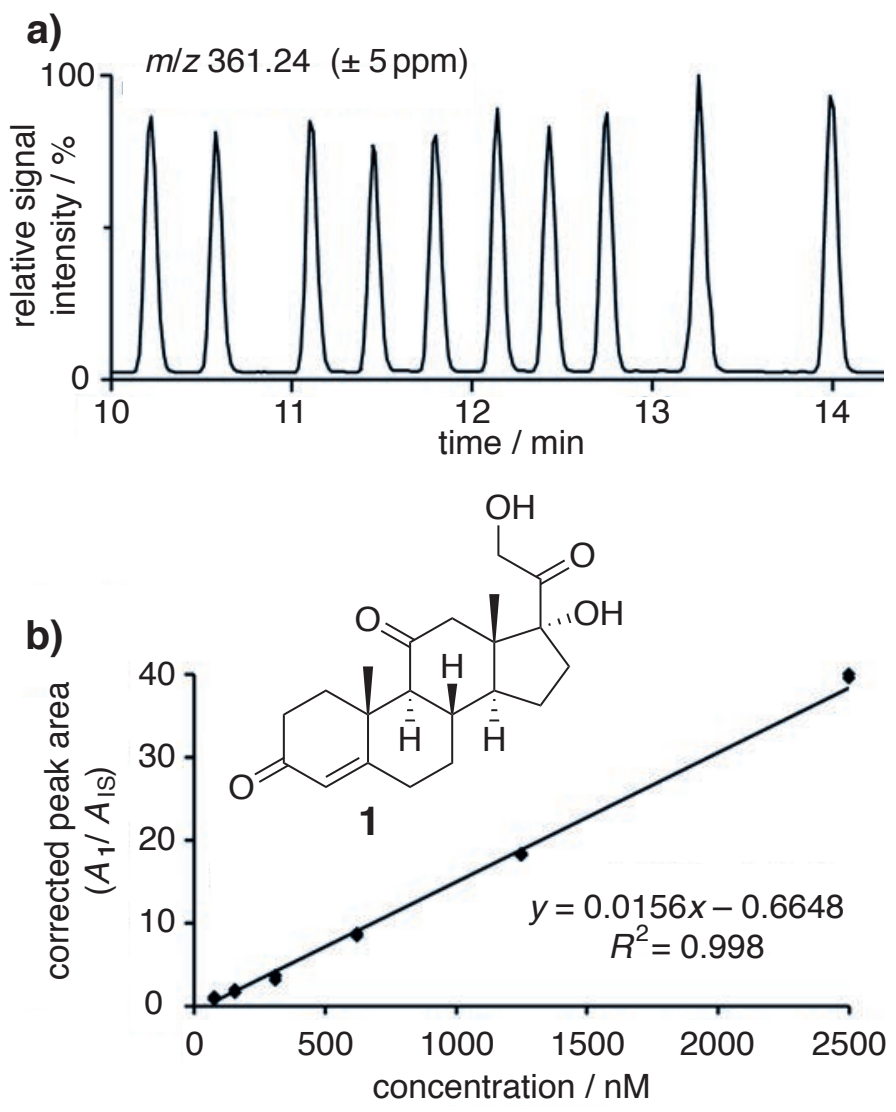

Fig. 2. FI characteristics; a) shows a series of $500 \mathrm{nM}$ cortisone (1) injections; b) illustrates a linear signal response in respect to the injected 1 concentration. Reprinted (adapted) with permission from ref. [18]. Copyright 2013 American Chemical Society. regime. This allows extraction within 5 min which results in a $\approx 15$ min cycle time including washing and conditioning of the solidphase. For demonstration purposes, the method was applied to extract compound $\mathbf{2}$ as well as oxazepam and diazepam from human plasma. Their limits of quantification were about $500 \mathrm{ng} \mathrm{ml}^{-1}$ and the relative standard deviation around $20 \%$.

In a follow-up study, Ghiasikhou et al. immobilized bovine carbonic anhydrase II (CAII) on the pin of the gap sampler using epoxy-modified beads followed by an epoxy-amine reaction. ${ }^{[22]}$ CAII can be used for specific extraction for sulfonamide bearing analytes which bind non-covalently with a high affinity. The bound analytes can be desorbed by immersion of the CAII modified pin into a 50:50 $\mathrm{ACN} / \mathrm{H}_{2} \mathrm{O} V / V$ carrier liquid as shown in Fig. 1e. To evaluate the extraction's specificity, acetazolamide (3), diazepam (4) and monomethyl auristatin E (5) were used as model compounds in PBS. In a first experiment, they were extracted with the epoxy-modified beads only. In a second experiment, the CAII-modified beads were used. As shown in Fig. 4, 330\% more sulfonamide-bearing $\mathbf{3}$ was extracted by the CAII phase. In contrast, sulfonamide-absent $\mathbf{3}$ and $\mathbf{4}$ were less efficiently extracted highlighting the specificity. No CAII deterioration was observed even after 40 extractions. Furthermore, the method was applied to analyze an anti-tumor small molecule-drug conjugate (SMDC) in a blood sample. The SMDC is composed of $\mathbf{3}$ and $\mathbf{5}$ derivatives joined by a valine-citrulline (ValCit) dipeptide linker and is referred to as 3-ValCit-5. The drug's mode of action is based on the specific binding of $\mathbf{3}$ to human CAIX which is overexpressed in many cancer cells. Subsequently, ValCit is cleaved by Cathepsin $\mathrm{B}$ and releases the cytotoxic structure 5.[23] Using the gap sampler for the specific extraction of 3-ValCit-5, the drug was extracted from a $2.5 \mu \mathrm{g} \mathrm{ml}^{-1}$ human plasma sample. Undoubtedly, this highlights that even complex samples can be analyzed in a very short time without manual sample preparation prior to ESI-MS.

\section{The Gap Sampler for Bioaffinity Screenings}

ESI-MS is a suitable technique to measure the bioaffinity of a small molecule towards a biological target, e.g. a protein. ${ }^{[24]}$ Under soft ionization and transfer conditions, the non-covalent protein-ligand complex can be detected directly via ESI-MS.[25] Thus, the gap sampler can be used as a high-throughput bioaffinity screening device. However, an aqueous carrier liquid is required to maintain the native protein structure. Modifications on the gap sampler's capillaries, as suggested by Ghiasikhou et al., made this possible. ${ }^{[26]}$ The drain and feed capillaries were replaced with one single capillary bearing an upwards oriented hole.

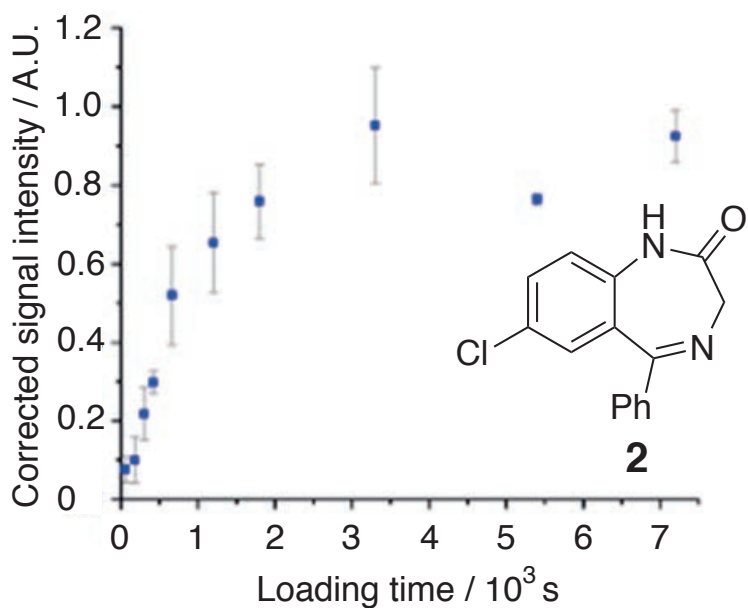

Fig. 3. Extraction characteristics of nordazepam (2) with the $C_{18}$ modified pin. Increasing the extraction time increases the observed signal until an equilibrium is reached. Reprinted (adapted) with permission from ref. [21]. Copyright 2017 Springer Nature. 


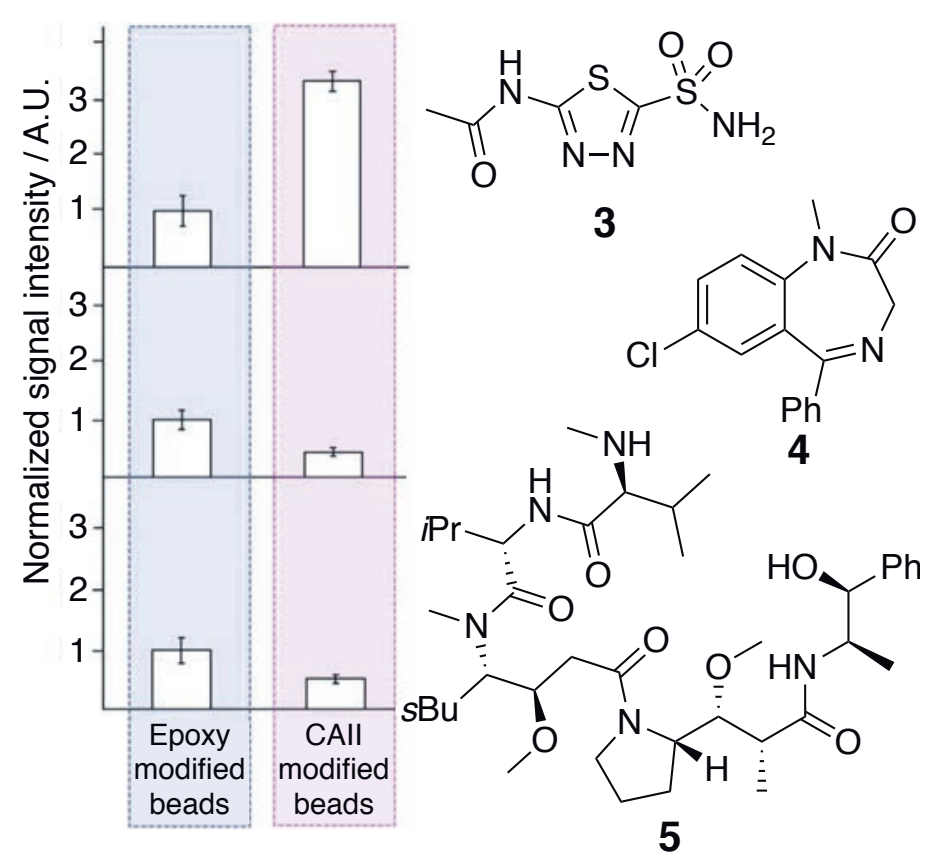

Fig. 4. Comparison between unspecific (blue, epoxy modified) and specific (magenta, CAll modified) extraction beads. Acetazolamide (3), diazepam (4) and monomethyl auristatin E (5) were extracted with both phases. Since CAll binds sulfonamides non-covalently, 3 is specifically enriched while there is no particular selectivity for $\mathbf{4}$ and $\mathbf{5}$. Reprinted (adapted) with permission from ref. [22]. Copyright 2019 Springer Nature.

Our current research focuses on implementing this new capillary for an automated bioaffinity screening. The concept is depicted in Fig. 1f. The carrier liquid contains a constant concentration of the protein target, while potential ligands are picked up from the multiwell plate and injected into the liquid bridge. In case of binding, the resulting non-covalent complex can be detected by ESI-MS. For a proof of concept experiment, CAII (P) was used as a model target. A $\mathbf{P}$ solution in $10 \mathrm{mM} \mathrm{NH}_{4} \mathrm{OAc}$ was fed into the capillary and a furosemide (6) solution was injected. A few seconds after injection, the complex $[\mathbf{P + 6}]$ was observed in the extracted ion chromatogram (XIC) of $[\mathbf{P}+\mathbf{6}]^{+10}$ as shown in Fig. 5a. Meanwhile, the unbound protein was depleted, i.e. the $\mathbf{P}^{+10}$ XIC decreased in Fig. 5b. Thus, the binding of the sulfonamidebearing compound 6 was confirmed. Likewise, Fig. $5 \mathrm{c}$ shows the spectrum before the complex was detected. Different charge states and acetate (Ac) adducts of $\mathbf{P}$ were observed. Fig. 5d shows the spectrum during complex detection when the dominant peaks appeared from $[\mathbf{P}+\mathbf{6}]$ complex species. If the concentrations of $\mathbf{P}$ and $\mathbf{6}$ are known during ESI, the binding affinity can be quantified based on the observed ratio of bound to unbound $\mathbf{P}$. Integrating all the adduct signals for all the charges states, a dissociation constant $K_{\mathrm{D}}=2.69 \mu \mathrm{M}$ was computed for the example in Fig. 5.[27] This compares well with values reported in literature for surface plasmon resonance $(\mathrm{SPR})$ data which determined $K_{\mathrm{D}}=2.38 \mu \mathrm{M}$. ${ }^{[28]}$ The exact protocols and the complete results, including a comparison with the 'gold standard' technique SPR, will be subject of an upcoming article.

\section{Conclusion: the Gap Sampler is a Versatile Autosampler}

Looking at the performance characteristics, the gap sampler can compete with the commercial and prototype systems in terms of cycle time and sample volumes. The pin-based sampling, the sample transfer and the FI into a carrier liquid with substantial mixing makes the autosampler relatively difficult to operate. However, this also opens opportunities for applications beyond the simple FI analysis. On the one hand, surface modification of

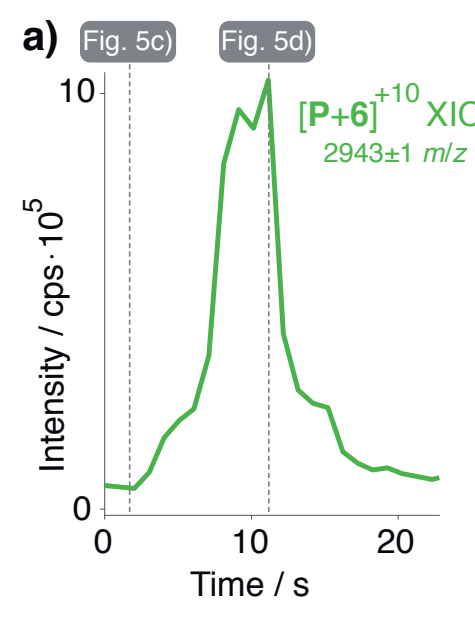

b) Fig. 5c) Fig. 5d)
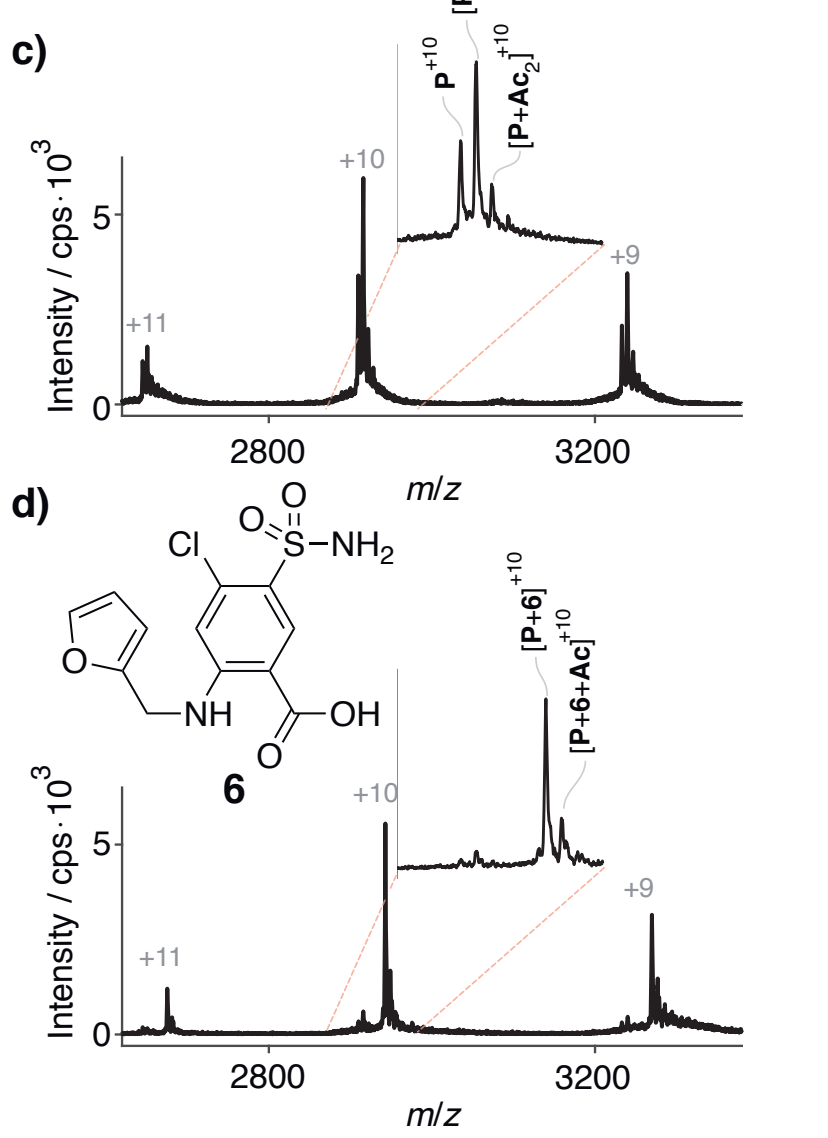

Fig. 5. Example of a bioaffinity screening where furosemide (6) is injected into a stream of CAll (P); a) shows the extracted ion chromatogram $(\mathrm{XIC})$ after injection for the complex $[\mathbf{P + 6}]^{+10}$ ion. The mass spectra presented in c) and d) correspond to the indicated time points; b) shows the XIC of the unbound $\mathbf{P}^{+10}$ ion; c) shows the spectrum before $\mathbf{6}$ passed through the system; d) shows the spectrum at the point where the most intense $[\mathbf{P + 6}]$ complex signals are detected.

the pin allows unspecific and specific extraction which is particularly useful in the fast and automated separation free analysis of complex samples. On the other hand, the flush-out characteristics inside the capillaries enable mixing of protein targets with ligands inside the ESI-capillary and thus open the possibility for bioaffinity screenings. Therefore, the major strengths of the gap sampler lie in its broad range of applications from FI over bioaffinity screenings to automated extractions.

\section{Outlook: What the Gap Sampler Could Analyze in the Future}

What has been learned from the gap sampler so far can be implemented in device modifications or novel applications in the 
future. For future hardware modifications, it might be advantageous to replace the overpressure chamber with a subatmospheric pressure region towards the MS. This would allow easier and faster sample deposition into the liquid bridge. In fact, other autosamplers rely on an aspiration, e.g. the mini DI sampler described by Felten et al. uses a subatmospheric chamber. ${ }^{[6]}$ This would allow sample injection without inserting the pin into the pressure chamber. Consequently, mechanical control of the pin would be easier and faster.

The full potential of the gap sampler has not been utilized yet and many future applications can be envisioned. Particular attention should be given to the gap sampler's characteristics and strengths, i.e. the pin-based sampling and the mixing characteristics during FI. Hence, the gap sampler could be used for surface sampling. For instance, the pin could pick up an extraction solvent followed by a solid-liquid extraction on top of a surface. Extractant absorption is a known problem when sampling from a wettable surface. ${ }^{[29]}$ However, the gap sampler might be ideal for sampling from a tissue section where the liquid extractant normally would be absorbed. For example, the pin could be preconditioned by a hot drying gas and then be held against a tissue section kept at $<0{ }^{\circ} \mathrm{C}$. The melted water containing analytes would be transferred for FI. Using the gap sampler's mixing characteristics during FI, another application might involve rapid derivatization of analytes inside the ESI-capillary. Similar to other studies, a cyanide-containing sample could be injected into a carrier liquid containing a dialdehyde reagent. ${ }^{[15 c]}$ This would allow the detection of the low mass cyanide analyte, for example in blood after intoxication, at increased sensitivity and specificity. Furthermore, the gap sampler's mixing characteristics could allow ligand exchange experiments when, for example, an inhibitor is injected into a stream of substrate incubated protein. Consequently, we could perform bioaffinity screenings based on reporter ligands or bioactivity screenings based on inhibition experiments.

\section{Acknowledgements}

The author especially wants to thank Prof. Renato Zenobi, Dr. Christof Fattinger, Dr. Adrien Marchand, Roger Steiner, Jonas Metternich, Martin Köhler, Lara Sutter, Alina Begley and Sahar Ghiasikhou for their help, input and comments. He is also grateful to the Scholarship Fund of the Swiss Chemical Industry (SSCI) for the financial support as well as to Metrohm AG and the Swiss Chemical Society for the best oral presentation award.

Received: February 14, 2020

[1] F. W. McLafferty, Science 1981, 214, 280, DOI: 10.1126/science.7280693.

[2] a) M. Yamashita, J. B. Fenn, J. Phys. Chem. 1984, 88, 4451, DOI: 10.1021/ j150664a002; b) C. Meng, M. Mann, J. Fenn, Zeitschr. Phys. D 1988, 10, 361, DOI: 10.1007/BF01384871.

[3] R. González-Domínguez, A. Sayago, Á. Fernández-Recamales, Bioanalysi 2017, 9, 131, DOI: 10.4155/bio-2016-0202.

[4] S. A. Hofstadler, K. A. Sannes-Lowery, Nat. Rev. Drug Discov. 2006, 5, 585 DOI: $10.1038 / \mathrm{nrd} 2083$.

[5] X. Cheng, J. Hochlowski, Anal. Chem. 2002, 74, 2679, DOI: 10.1021/ ac020186f.

[6] C. Felten, F. Foret, M. Minarik, W. Goetzinger, B. Karger, Anal. Chem 2001, 73, 1449, DOI: 10.1021/ac001074w.

[7] J. Šesták, D. Moravcová, V. Kahle, J. Chromatogr. A 2015, 1421, 2, DOI 10.1016/j.chroma.2015.07.090.

[8] T. Wang, L. Zeng, T. Strader, L. Burton, D. B. Kassel, Rapid Commun. Mass Spectrom. 1998, 12, 1123, DOI: 10.1002/(SICI)10970231(19980831)12:16<1123::AID-RCM293>3.0.CO;2-W.
[9] J. Pei, Q. Li, R. T. Kennedy, J. Am. Soc. Mass Spectrom. 2010, 21, 1107, DOI: $10.1016 /$ j.jasms.2010.02.013.

[10] D. Q. Jin, Y. Zhu, Q. Fang, Anal. Chem. 2014, 86, 10796, DOI: 10.1021/ ac503014k.

[11] C. Van Pelt, S. Zhang, J. Henion, J. Biomol. Techn. JBT 2002, 13, 72.

[12] G. A. Schultz, T. N. Corso, S. J. Prosser, S. Zhang, Anal. Chem. 2000, 72, 4058, DOI: 10.1021/ac000325y.

[13] C. K. Van Pelt, S. Zhang, E. Fung, I. Chu, T. Liu, C. Li, W. A. Korfmacher, J. Henion, Rapid Commun. Mass Spectrom. 2003, 17, 1573, DOI: 10.1002/ rem. 1087.

[14] C. C. Özbal, W. A. LaMarr, J. R. Linton, D. F. Green, A. Katz, T. B. Morrison, C. J. Brenan, Assay Drug Dev. Technol. 2004, 2, 373, DOI: 10.1089/adt.2004.2.373

[15] a) K. Hiraoka, K. Nishidate, K. Mori, D. Asakawa, S. Suzuki, Rapid Commun. Mass Spectrom. 2007, 21, 3139, DOI: 10.1002/rcm.3201; b) L. C. Chen, K. Nishidate, Y. Saito, K. Mori, D. Asakawa, S. Takeda, T. Kubota, H. Hori, K. Hiraoka, J. Phys. Chem. B 2008, 112, 11164, DOI: 10.1021/jp803730x; c) T. Murata, K. Zaitsu, in 'Ambient Ionization Mass Spectrometry in Life Sciences', Elsevier, 2020, p. 171, DOI: 10.1016/ B978-0-12-817220-9.00006-0; d) Z. Chen, S. Yamaki, J. Dong, Y. Hashi, H. Naoki, K. Eberhardt, 'Fast screening of pesticides in foods and agricultural products with probe electrospray ionization (PESI) tandem mass spectrometry', www.ssi.shimadzu.com/sites/ssi.shimadzu.com/files/Industry/ Literature/AOAC2019/P-W-043-AOAC-2019-fast-pesticides-PESI.pdf, accessed 28.01.2020.

[16] I. Sinclair, M. Bachman, D. Addison, M. Rohman, D. C. Murray, G. Davies, E. Mouchet, M. E. Tonge, R. G. Stearns, L. Ghislain, Anal. Chem. 2019, 91, 3790, DOI: 10.1021/acs analchem.9b00142.

[17] P. Berndt, C. Fattinger, R. Steiner, US 9446408 B2, 2016.

[18] V. Neu, R. Steiner, S. Muller, C. Fattinger, R. Zenobi, Anal. Chem. 2013, 85, 4628, DOI: $10.1021 / \mathrm{ac} 400186 \mathrm{t}$.

[19] V. Neu, P. Dörig, C. Fattinger, S. Müller, R. Zenobi, Microfluid. Nanofluid. 2016, 20, DOI: 10.1007/s10404-016-1732-3.

[20] N. Reyes-Garces, E. Gionfriddo, G. A. Gómez-Ríos, M. N. Alam, E. Boyacı, B. Bojko, V. Singh, J. Grandy, J. Pawliszyn, Anal. Chem. 2017, 90, 302, DOI: 10.1021/acs.analchem.7b04502.

[21] S. Ghiasikhou, M. F. da Silva, Y. Zhu, R. Zenobi, Anal. Bioanal. Chem. 2017, 409, 6873, DOI: 10.1007/s00216-017-0652-8.

[22] S. Ghiasikhou, S. Cazzamalli, J. Scheuermann, D. Neri, R. Zenobi, Anal. Bioanal. Chem. 2019, 411, 7387, DOI: 10.1007/s00216-019-02165-7.

[23] a) G. M. Dubowchik, R. A. Firestone, L. Padilla, D. Willner, S. J. Hofstead, K. Mosure, J. O. Knipe, S. J. Lasch, P. A. Trail, Bioconj. Chem. 2002, 13, 855, DOI: 10.1021/bc025536j; b) S. Cazzamalli, A. Dal Corso, D. Neri, J. Controll. Rel. 2017, 246, 39, DOI: 10.1016/j.jconrel.2016.11.023.

[24] J. Kool, M. Giera, H. Irth, W. M. Niessen, Anal. Bioanal. Chem. 2011, 399, 2655, DOI: $10.1007 / \mathrm{s} 00216-010-4406-0$.

[25] J. A. Loo, Mass Spectrom. Rev. 1997, 16, 1, DOI: 10.1002/(SICI)10982787(1997)16:1<1::AID-MAS1>3.0.CO;2-L

[26] S. Ghiasikhou, A. Marchand, R. Zenobi, Microfluid. Nanofluid. 2019, 23, 60, DOI: 10.1007/s10404-019-2229-7.

[27] A. F. Gavriilidou, B. Gulbakan, R. Zenobi, Anal. Chem. 2015, 87, 10378, DOI: $10.1021 /$ acs.analchem.5b02478.

[28] M. C. Jecklin, S. Schauer, C. E. Dumelin, R. Zenobi, J. Mol. Recognit. 2009, 22, 319, DOI: 10.1002/jmr.951.

[29] M. J. Walworth, J. J. Stankovich, G. J. Van Berkel, M. Schulz, S. Minarik, J. Nichols, E. Reich, Anal. Chem. 2010, 83, 591, DOI: 10.1021/ac102634e.

\section{License and Terms}

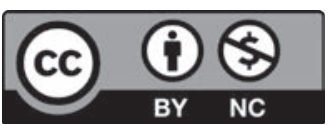

This is an Open Access article under the terms of the Creative Commons Attribution License CC BY_NC 4.0. The material may not be used for commercial purposes.

The license is subject to the CHIMIA terms and conditions: (http:// chimia.ch/component/sppagebuilder/?view = page\&id=12).

The definitive version of this article is the electronic one that can be found at doi:10.2533/chimia.2020.220 